\title{
Temperopareital Methyl Methacrylate Cranioplasty-A Preliminary Case Study And Technical Note
}

\author{
Dr.P.Subramanian MDS^, Dr.Vidya Albert Yen,MDS, ${ }^{*}$ Drthirupathyms Mch ^ \\ ${ }^{\wedge}$ Scraniofacial And Neurosurgery Unit, Abchospital ,Trichirapalli \\ *Associate Professor, Chengalpattu Medical College \& Hospital
}

\begin{abstract}
Temperoparietalcranioplasty are largely performed among all other varieties of craniolplasty and the role of Dental surgeon in these case is discussed in elaborate in restoring the defect with polymethyl methacrylate plate Post trauma cranial defects in 5 selected cases and their defect correction technique discussed in this paper.
\end{abstract}

\section{Introduction}

Cranioplasty is defined as the repair of a cranial defect or deformity. It replaces the natural protective structures of the brain and restores the shape and appearance of cranium.Various techniques and materials have been used to repair cranial defects, such as autogenous bone grafts and alloplastic implants. We routinely use methyl methacrylate as a cranioplastic material because it is relatively cheap,reliable and easily available.

Cranial vault deformities are usually due to the result of trauma ,tumor ablation, or congenital abnormalities. Major indications of cranioplasty are mechanical protection of the brain from trauma and restore esthetic contour

Cranioplasty is the surgical intervention of cranial defects dates back 7000BC.Archeologic findings proved that the use of inorganic materials for cranioplasty had begun before the organic materials. In $19^{\text {th }}$ century, the use of bone from different donor sites such as ribs,tibia gained popularity. but there is still no better consenses achieved

An ideal cranioplasty material must have the features like better fit, radiolucency,resistance to infections, very low heat conduction,strong biomechanical properties, non magnetic,biocompatible,easy to mould,less expensive and readily available. Perfection in cranioplasty is uphill and ongoing research on biologic and nonbiologic substitutions continues. Stem cell experiments and development of morphogenic proteins are expected to bring some change in future.

\section{History}

In World War 2 there was large need for cranioplasty and acrylic was used by dentist $\mathrm{s}$ with ease. Acrylic in the form of methyl methacrylate \{polymethylmethacrylate \} was first used in animal models and then used in Humans widely after the study of Spence in 1954. Cold cure material was first used and the heat evolved due to the exothermic reactions as well increased amount of residual monomer causes granulation tissue formation and reactions to the soft tissues. Even now the accurate fit with cold cure resins attracts and inclines many surgeons to it and the troublesome prosthetic procedures are avoided.

We stress the need for Heat cure material to be used which gives good strength, durability and negligible residual monomer toxicity. There may be some inaccuracy in fit of margins but that can be repaired with rapid cold cure material if required intraoperatively.

Moreover the pore size in heat cure acrylic resins is approximately more than $100 \mathrm{u}$ microns which aid in bone ingrowth and relatively a inert material well tolerated.

Cranioplasty after post injury decompressivecraniectomy is routine nowadays as there is increase in incidence of high velocity injury and appropriate timing of intervention is not clear. Potential benefits from delayed intervention $\{>6$ weeks $\}$ for reducing the infection risk must be balanced by persistent altered CSF dynamics to hydrocephalus. Many studies indicate no specific time frame was predictive of hydrocephalus or infection and logistic regression to identify significant predictors among the collected variables. Some authors claim immediate reconstruction reducing the cost and fast rehab but others inclined to late procedures only .Out come of either methods are the same and no significant advantages noted other than logistics.

This article presents a clinical report in which an inexpensive method was used to fabricate a preformed heat cured acrylic resin cranial prosthesis to rehabilitate tempero-pareital defect with success 


\section{Clinical report}

From the month of August to October 2010, 5 cases of temperopareitalcranioplasty was selected for Acrylic cranioplasty. All the cases were post trauma craniectomy age ranges from 35-40 years, male patients. All the patients were 9 months to 1year post op after initial craniectomy. All of them had communitedtemperopareital bone injury and extreme bone loss was present beyond preservation. All the patients had good neurological recovery and were ambulatory with perseverance of Pre trauma life style. Hence the Aim of the surgery was repair of cranial defect and esthetics. The size of the defects varied from 10x $6 \mathrm{~cm}---12 \times 6.5 \mathrm{~cm}$ with good non scary scalp present.

\section{Procedure}

Proper identification of defect in the surgical site was done by digital palpation and defect extent was marked with marker. CT scan can be used to identify the location of defect margins under temporalis cover and cl;inically confirmed.
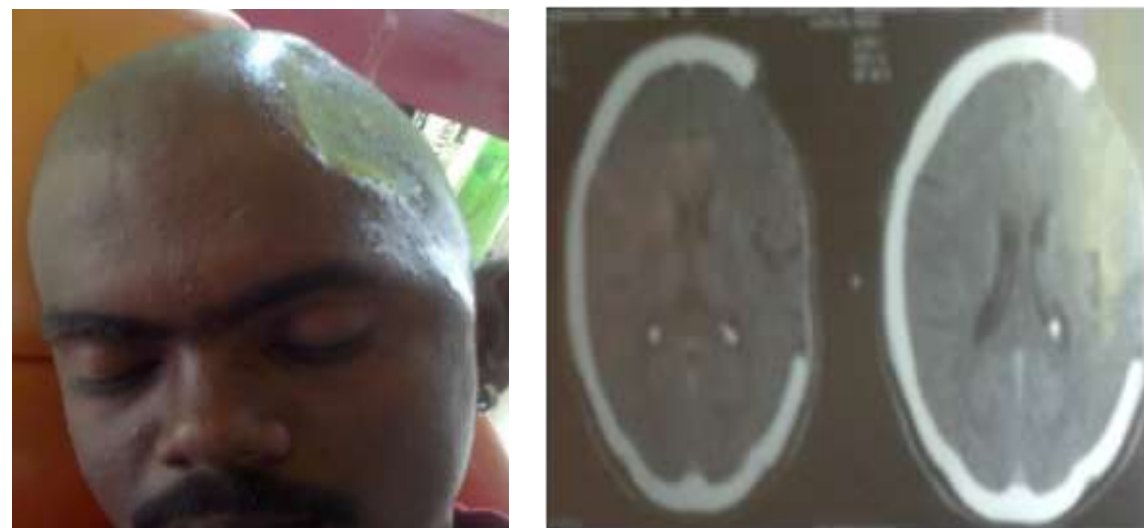

Defect
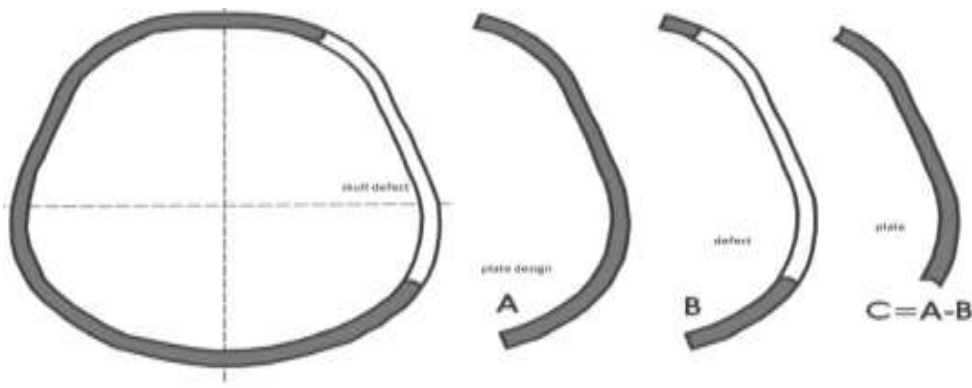

After locating the margins of the defect the plate is planned $0.5-1 \mathrm{~cm}$ oversize to facilitate the trimming and fit of prosthesis in the intraoperative maneuver. Hair in the site is cleared with liberty as the patients are males and Vaseline is applied to the skin Ear holes are plugged with gauze and eye antibiotic ointment with gauze shield secured with micropore plaster tape. Head placed in lateral position in supine postion to facilitate impression . Impression technique
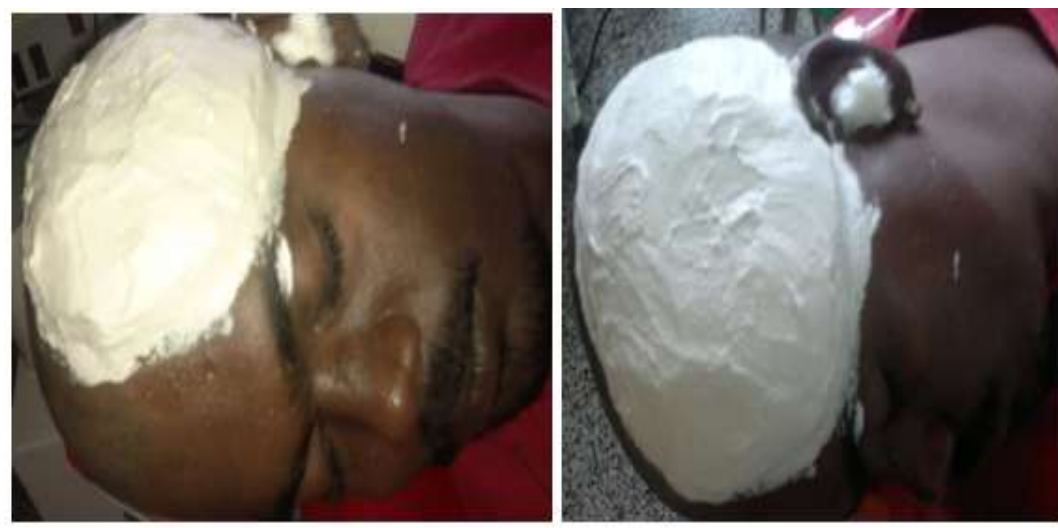
A modeling soft wax sheet \{approximately 1 1/2sheet thickness \} is adapted to the defect margins marked and secured well.A micropore adhesive tape or light body elastic impression material can be coated for securing adaptation. Plaster of paris is mixed in dough consistency and poured over the wax sheet covering the entire defect and impression is made. The impression is then transferred to a surgical instrument tray filled with doughy plaster of paris as shown and allowed to set .

Another surgical tray of same size is taken and negative impression by pouring plaster of paris is done to complete the modeling process. After setting the trays were separated and wax sheet is removed with ease which is acting as a spacer. The models gently polished with sand paper and smooth surfaces are obtained without distorting. Small repairs of defects can also be carried out with plaster of paris to correct the unevenness noted if any.
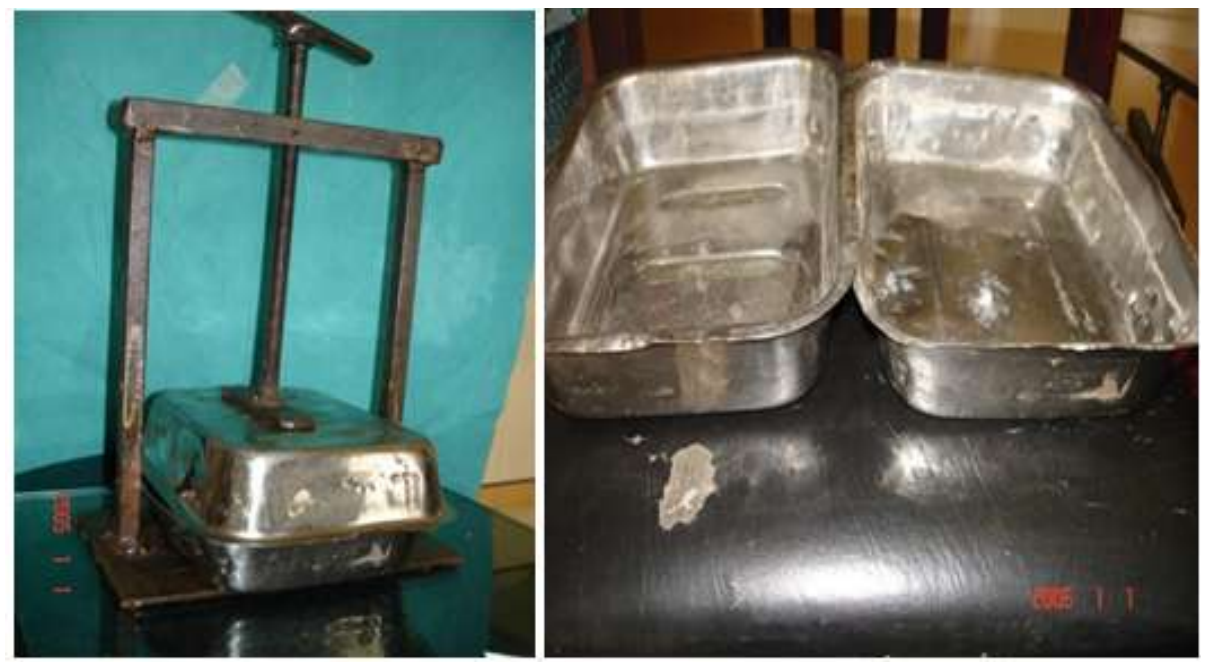

trays and clamps

Now a routine heat cure acrylic processing is carried out and material is packed and curing cycle for 1 hour is advised. After curing cycle, bench cooling was completed and the prosthesis removed carefully .Try to preserve the plaster moulds as sometimes they are useful in intra operative corrections and repair.

Polishing of prosthesis is done with fine emery sheet /sandpaper and clean water only. Acrylic trimmers are used to take out residual plaster and one has to ensure no contaminates present on the plate. You can use a magnifying glass in a well lit area to identify minute debris and clean properly. Polishing agents/ chemicals/wax etc should be avoided as the residue will cause reaction on tissues. Prosthesis should be thoroughly brushed in lathe and micro debris cleared out of it. It has to be rinsed in running water for minimum 20 minutes after final polishing and soaked in betadaine antiseptic solution for 30 minutes .Then the prosthesis cleaned of betadaine thoroughly and packed in sterilizing cover to gamma irradiation. The plaster moulds also washed well and sent for gamma irradiation to be used in theatre for assisting correction and repair with self cure material.

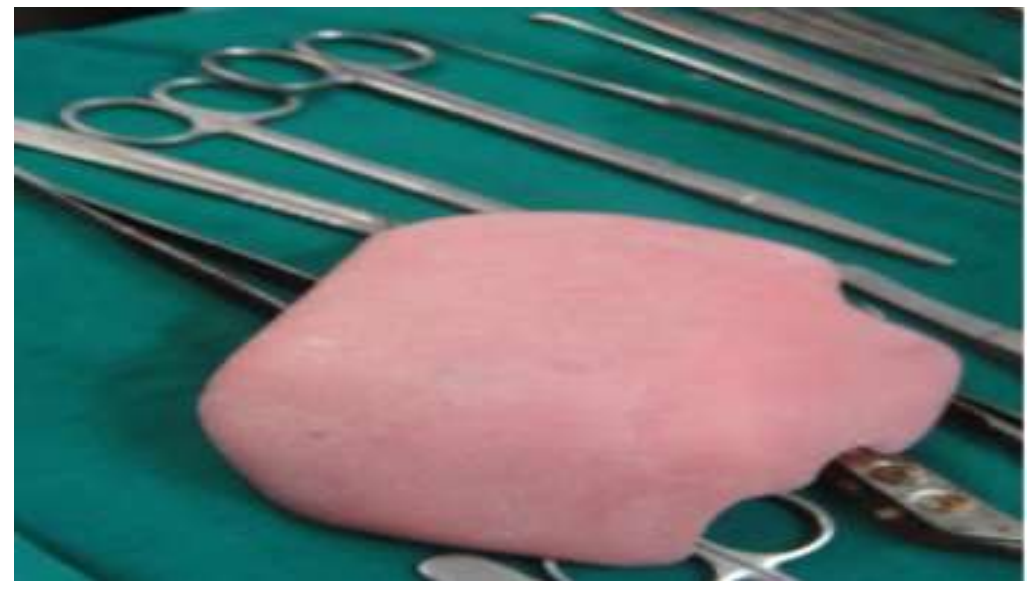

Trimming acrylic plate 


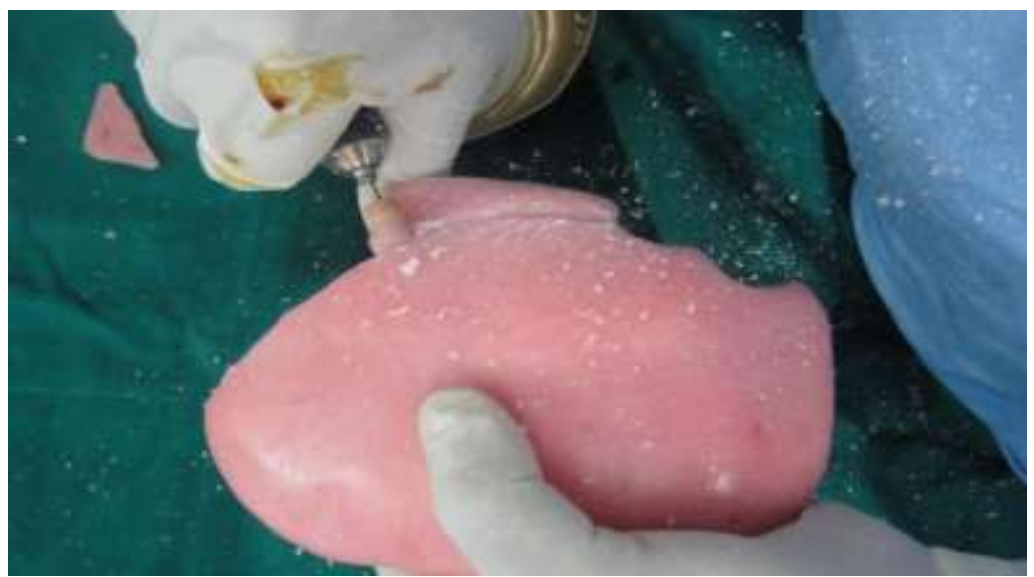

Intraoperatively the prosthesis is once again washed in normal saline and diluted betadaine before preliminary trial .The trimming process and acrylic works should be carried out well at least 6metres away from the patient as the microdebris/dust can contaminate the wound. Brain tissue can be covered with sterile cellophane paper and trial can be done. After final try in, the prosthesis is polished with smooth acrylic trimmers and rough ends are polished. The plates should undergo thorough washing process to remove acrylic dust before placement.

Prosthesis is secured in position with dovetail fitting and 3 large $\mathrm{x}$ shaped $1.5 \mathrm{~mm}$ titanium plates and $8 \mathrm{~mm}$ screws to prevent rotation. And wound closed away from the margins of the plate. A layer of pericranium is sutured before scalp for additional protection. Drains placed before closure to avoid seroma collection. Electrosugery allows lot of inflammatory collection hence minimal use of cautery is advised with adequate irrigations with coolants.

Post op care is routine and wound care is followed with parentral antibiotic cover for a minimum of 7 days. Wound sutures are removed after 6 days and patient discharged.

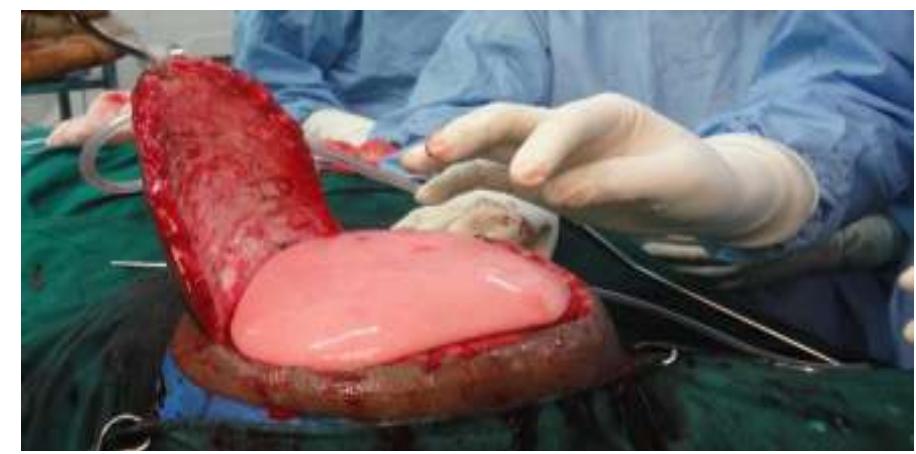

Intraop

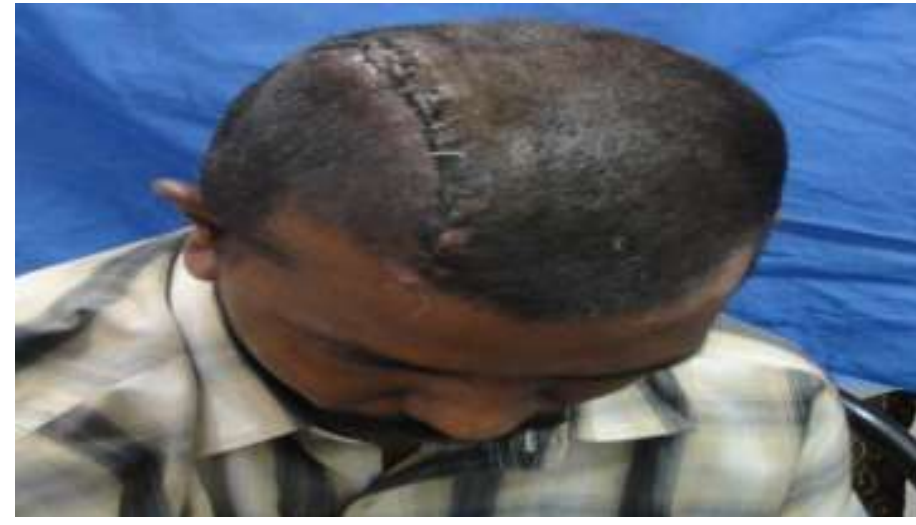

postop

The common postop complaints are headache; tenderness in the surgical site is managed by analgesics One patient had prolonged seroma collection and aspiration had to be made in the follow up for a period of 2 
months. Tenderness was present in margins of wound for more than 3 months in 2 cases. Wound healing was good in all the cases and post operative wound complications are not seen. Post operative CT scan reveals no abnormalities in the reconstructed area. Plate sinking, cutthro, dehiscence, rotation,mobility, infection were not noted. Mild over contouring in the margins and slight elevation noted after wound shrinkage in 2 cases..Hair growth camouflaged these discrepancies in both the cases.

The mean follow up in these cases were 13 months. Intraoperative impression techniques is planned in our next study with hopes of good precision in the beveled margin fit will be improved in our next study group. It has to be noted that the thickness of scalp flap in impression procedure and wax/plaster mould preparation compensates well in all the cases during placement over mildly altered swollen brain tissue.Adaptation to the contour of defect is good.

\section{Conclusion}

Acrylic cranioplasty is a versatile modality in correcting cranial defects and the outcome is desirable and good as reported by many authors. Though many methods are available to do the prosthesis but authors found the above mentioned technique is simple and can be practiced with ease.

\section{References}

[1]. Abdulah AE et al Cranioplasty using polymethylmethaacrylate Ghana med journ 2006;40;18-21

[2]. BeumerJ et al maxillofacial rehabilitation, Toronto St Louis London 1979;372-97

[3]. Communication AuthorsProf DrP Subramanian MDS Oral and maxillofacial surgeon drsubbu@ gmail.com 